\title{
ON MEAN VALUES
}

J. ACZÉL

Introduction. Historical. In 1930 Kolmogoroff and Nagumo ${ }^{1}$ proved simultaneously a fundamental theorem on mean values. In their definition a mean value is an infinite sequence of functions: $M_{1}\left(x_{1}\right)=x_{1}, M_{2}\left(x_{1}, x_{2}\right), M_{3}\left(x_{1}, x_{2}, x_{3}\right), \cdots, M_{n}\left(x_{1}, \cdots, x_{n}\right), \cdots$ Each function of this sequence has to satisfy the following conditions: $M_{n}(x, \cdots, x)=x, M_{n}\left(x_{1}, \cdots, x_{n}\right)$ must be a continuous, (strictly) increasing (cf. \$2) and symmetric function. The terms of this sequence are connected by the "associative property": $\left(M_{k}=M_{k}\left(x_{1}, \cdots, x_{k}\right)\right)$

$$
\begin{array}{r}
M_{n}\left(x_{1}, \cdots, x_{k}, x_{k+1}, \cdots, x_{n}\right)=M_{n}\left(M_{k}, \cdots, M_{k}, x_{k+1}, \cdots, x_{n}\right) \\
(k \leqq n=1,2,3, \cdots) .
\end{array}
$$

The theorem of Kolmogoroff and Nagumo is that these conditions are necessary and sufficient for the existence of a continuous and (strictly) increasing function $f(x)$ by which the mean value can be written in the form $\left(f^{-1}(x)\right.$ is the inverse function of $\left.f(x)\right)$ :

(1) $M_{n}\left(x_{1}, \cdots, x_{n}\right)=f^{-1}\left(\frac{f\left(x_{1}\right)+\cdots+f\left(x_{n}\right)}{n}\right)(n=1,2,3, \cdots)$.

In the next year de Finetti and B $\not$ rge Jessen ${ }^{2}$ extended this theorem for mean values of functions. De Finetti and Kitagawa ${ }^{8}$ considered weighted means where besides the variables $x_{1}, x_{2}, \cdots, x_{n}$ also the weights $q_{1}, q_{2}, \cdots, q_{n}\left(q_{1}+q_{2}+\cdots+q_{n}=1\right)$ were given and gave the conditions for the possibility of writing them in the form

$$
\begin{array}{r}
M_{n}\left(x_{1}, \cdots, x_{n} ; q_{1}, \cdots, q_{n}\right)=f^{-1}\left[q_{1} f\left(x_{1}\right)+\cdots+q_{n} f\left(x_{n}\right)\right] \\
(n=1,2,3, \cdots)
\end{array}
$$

analogous to (1) $\left(q_{1}+\cdots+q_{n}=1\right)$.

Received by the editors July 16, 1947.

1 A. Kolmogoroff, Sur la notion de la moyenne, Atti della R. Academia nazionale dei Lincei (6) vol. 12 (1930). M. Nagumo, Utber eine Klasse der Mittelwerte, Jap. J. Math. vol. 7 (1930).

${ }^{2}$ B. de Finetti, Sul concetto di media, Giornale di Istituto Italiano di Attuarii vol. 2 (1931). B. Jessen, Bemaerkinger om konvekse Funktioner og Uligheder imellem Middelvaerdier, I; II, Matematisk Tidsskrift B (1931). Cf. also J. Horvath, On a theorem of Børge Jessen, Norske Videnskabers Selskabs Forhandlinger vol. 20 (1947).

${ }^{3} \mathrm{~T}$. Kitagawa, On some class of weighted means, Proceedings of the Physico-Mathematical Society of Japan vol. 16 (1934). 
Conditions by which a mean value defined for one definite number of variables can be written in the form (1) were posed first by Aumann ${ }^{4}$ for the case when $M\left(x_{1}, \cdots, x_{n}\right)$ is an analytic function. His proof uses rather intricate considerations.

In $\$ 1$ I intend to give necessary and sufficient conditions for the validity of (1) for means of one definite number of variables without supposing analyticity of $M\left(x_{1}, \cdots, x_{n}\right)$, only continuity and strict monotony as before. The property which stands instead of Kolmogoroff and Nagumo's "associativity" will be the "bisymmetry." This asserts that the function of $n^{2}$ variables

$$
\begin{aligned}
\psi_{2}\left(x_{11}, \cdots, x_{1 n} ; \cdots ;\right. & \left.x_{n 1}, \cdots, x_{n n}\right) \\
& =M\left[M\left(x_{11}, \cdots, x_{1 n}\right), \cdots, M\left(x_{n 1}, \cdots, x_{n n}\right)\right]
\end{aligned}
$$

does not alter if we replace $x_{i k}$ by $x_{k i}$ and vice versa.

In \$2 I show that if we drop the condition of symmetry the mean has the form (2); that is, without giving the weights we shall have the conditions by which a nonsymmetric mean is a weighted "Kolmogoroff-Nagumo mean." In \$3 I try to show the importance of the condition "bisymmetry," for by dropping also the condition $M(x, \cdots, x)=x$ ("reflexivity"), that is, by considering (continuous, increasing and) bisymmetric functions $\left[x_{1}, \cdots, x_{n}\right]$ which are not means any more, we shall see that they have the form

$$
\left[x_{1}, \cdots, x_{n}\right]=f^{-1}\left\{p_{1} f\left(x_{1}\right)+\cdots+p_{n} f\left(x_{n}\right)+p\right\} .
$$

To simplify our considerations we shall confine ourselves to mean values defined for two variables. This will mean no loss of generality. ${ }^{5}$

\section{Symmetric means.}

Definitions. We postulate the single-valued function of two variables $M(x, y), \alpha \leqq x, y \leqq \beta$ (definitions and theorem can be extended without difficulty for open or half open intervals which can be infinite as well), to fulfill the following conditions:

(i) Strict monotony: if $x<x^{\prime}$ then $M(x, y)<M\left(x^{\prime}, y\right)$ and the same for $y<y^{\prime}$;

(ii) Continuity;

${ }^{4}$ G. Aumann, Aufbau von Mittelwerten mehrerer Argumente, II. Analytische Mittelwerte, Math. Ann. vol. 110 (1935).

5 For a detailed discussion in the general case of $\boldsymbol{n}$ variables for symmetric means, cf. J. Aczél, The notion of mean values, Norske Videnskabers Selskabs Forhandlinger vol. 19 (1946).-For special simplifications in the case $n=2$ see J. Aczel, On mean values and operations defined for two variables, Norske Videnskabers Selskabs Forhandlinger vol. 20 (1947). 
(iii) Bisymmetry: $\psi_{2}\left(x_{11}, x_{12}, x_{21}, x_{22}\right)=M\left[M\left(x_{11}, x_{12}\right), M\left(x_{21}, x_{22}\right)\right]$ $=M\left[M\left(x_{11} x_{21}\right), M\left(x_{12}, x_{22}\right)\right]$;

(iv) Reflexivity: $M(x, x)=x$;

(v) Symmetry: $M(x, y)=M(y, x)$.

(i) and (iv) imply $x<M(x, y)<y$ if $x<y$ ("internness").

Generalizing (iii) we can define the "k-symmetry." We consider the following sequence of functions:

$$
\begin{aligned}
& \psi_{1}\left(x_{1}, x_{2}\right)=M\left(x_{1}, x_{2}\right), \\
& \psi_{2}\left(x_{11}, x_{12}, x_{21}, x_{22}\right)=M\left[M\left(x_{11}, x_{12}\right), M\left(x_{21}, x_{22}\right)\right], \\
& \psi_{8}\left(x_{111}, x_{112}, x_{121}, x_{122}, x_{211}, x_{212}, x_{221}, x_{222}\right) \\
& \quad=M\left\{M\left[M\left(x_{111}, x_{112}\right), M\left(x_{121}, x_{122}\right)\right], M\left[M\left(x_{211}, x_{212}\right), M\left(x_{221}, x_{222}\right)\right]\right\},
\end{aligned}
$$

( $\psi_{k}$ is a function of $2^{k}$ variables). $M(x, y)$ is " $k$-symmetric" if $\psi_{k}$ does not alter by changing variables, the indices of which are permutations of each other. By applying (iii) repeatedly we see that every bisymmetric function is k-symmetric.

We call $M(x, y)$ " $k$-symmetric in the stronger sense" if the function $\psi_{k}$ is symmetric in its $2^{k}$ variables. We can see that every symmetric and bisymmetric function is $k$-symmetric in the stronger sense.

Theorem. Conditions (i), (ii), (iii), (iv), (v) are necessary and sufficient for the existence of an increasing and continuous function $f(x)(\alpha \leqq x \leqq \beta)$ by which $M(x, y)$ has the form

$$
M(x, y)=f^{-1}\left(\frac{f(x)+f(y)}{2}\right) .
$$

The necessity of the conditions is evident. We prove the sufficiency by constructing $f(x)$ resp. its inverse function $\phi(x)=f^{-1}(x)$. This function has to be increasing, continuous and has to satisfy the functional equation $(u=f(x), v=f(y))$ :

$$
M[\phi(u), \phi(v)]=\phi\left(\frac{u+v}{2}\right) .
$$

We define $\phi(x)$ for the dyadic fractions as follows:

$$
\begin{gathered}
\phi(0)=r_{0}^{(0)}=\alpha, \quad \phi(1)=r_{1}^{(0)}=\beta ; \\
\phi(0)=r_{0}^{(1)}=M(\alpha, \alpha)=\alpha, \quad \phi(1 / 2)=r_{1}^{(1)}=M(\alpha, \beta), \\
\phi(1)=r_{2}^{(1)}=M(\beta, \beta)=\beta ;
\end{gathered}
$$




$$
\cdots, \phi\left(\frac{2 q}{2^{k+1}}\right)=r_{2 q}^{(k+1)}=M\left(r_{q}^{(k)}, r_{q}^{(k)}\right)=r_{q}^{(k)},
$$

$$
\phi\left(\frac{2 q+1}{2^{k+1}}\right)=r_{2 q+1}^{(k+1)}=M\left(r_{q}^{(k)}, r_{q+1}^{(k)}\right), \cdots ;
$$

$\phi(x)$ is increasing in consequence of (i).

Substituting the recursive formula (6) repeatedly in the expression of $\gamma_{p}^{k+1}$ we get finally a $\psi_{k+1}$ in which only $\beta$ 's and $\alpha$ 's figure as variables. We assert that the number of $\beta$ 's is exactly $p$. This can be proved by induction, because it is true for $k=0,1$ and if we suppose that in the $\psi_{k}$ representation of $r_{q}^{(k)}$ the number of $\beta$ 's is $q$, then, for example, in $r_{2 q+1}^{(k)}=M\left(r_{q}^{(k)}, r_{q+1}^{(k)}\right)$ this number must be $2 q+1$. Similarly in the $\psi_{k+1}$ of $M\left(r_{\ell_{1}}^{(k)}, r_{\ell_{2}}^{(k)}\right)$ the number of $\beta$ 's is $q_{1}+q_{2}$. And so it follows from the " $k$-symmetry in the stronger sense" that $M\left(r_{a_{1}}^{(k)}, r_{q_{2}}^{(k)}\right)=M\left(r_{a_{1}^{\prime}}^{(k)}, r_{a_{2}^{\prime}}^{(k)}\right)$ if $q_{1}+q_{2}=q_{1}^{\prime}+q_{2}^{\prime}$. Especially if $q_{1}+q_{2}$ $=2 q+s(s=0$ or 1$)$ then $M\left(r_{Q_{1}}^{(k)}, r_{Q_{2}}^{(k)}\right)=r_{2 \ell+s}^{k+1}$.

This enables us to show that for our dyadic fractions, $\phi(x)$ satisfies the functional equation (5). In fact, if we consider $u=q_{1} / 2^{k}, v$ $=q_{2} / 2^{k}\left(q_{1}+q_{2}=2 q+s ; s=0\right.$ or 1$)(u+v) / 2=(2 q+s) / 2^{k+1}$,

$$
\begin{aligned}
M[\phi(u), \phi(v)] & =M\left[\phi\left(\frac{q_{1}}{2^{k}}\right), \phi\left(\frac{q_{2}}{2^{k}}\right)\right]=M\left(r_{q_{1}}^{(k)}, r_{q_{2}}^{(k)}\right) \\
& =M\left(r_{q+8}^{(k)}, r_{q}^{(k)}\right)=r_{2 q+\varepsilon}^{(k+1)}=\phi\left(\frac{2 q+s}{2^{k+1}}\right)=\phi\left(\frac{u+v}{2}\right) .
\end{aligned}
$$

As the dyadic fractions are everywhere dense in the interval $(0,1)$ and $\phi(x)$ is monotonous on this set there exists a right and a left limit in every point of the interval $(0,1)$. We have to prove that they can not be different. For suppose on the contrary $y_{1}=\phi(x-0)<\phi(x+0)$ $=y_{2}$ then by the "internness" $y_{1}<M=M\left(y_{1}, y_{2}\right)<y_{2}$ and we can choose $\epsilon$ so that

$$
y_{1}+\epsilon<M<y_{2}-\epsilon .
$$

By (ii) there exists a $\delta$ for which whenever $\left|y_{1}-y_{1}^{\prime}\right|<\delta,\left|y_{2}-y_{2}^{\prime}\right|<\delta$

$$
M-\epsilon<M^{\prime}=M\left(y_{1}^{\prime}, y_{2}^{\prime}\right)<M+\epsilon \text {. }
$$

We can choose $y_{1}^{\prime}=\phi\left(q_{1} / 2^{k}\right), y_{2}^{\prime}=\phi\left(q_{2} / 2^{k}\right)$ with $z=\left(q_{1}+q_{2}\right) / 2^{k+1}>x$,

$$
\begin{aligned}
\phi(z) & =M\left[\phi\left(q_{1} / 2^{k}\right), \phi\left(q_{2} / 2^{k}\right)\right]=M\left(y_{1}^{\prime}, y_{2}^{\prime}\right) \\
& =M^{\prime}<M+\epsilon<y_{2}=\phi(x+0),
\end{aligned}
$$


by (7), (8), (9) in contradiction with the monotony of $\phi(x)$ and thus $\phi(x)$ is continuous. It follows immediately that $\phi(x)$ satisfies (5) in every point $0 \leqq x \leqq 1$ and so we constructed $\phi(x)=f^{-1}(x)$ and thus our theorem is proved.

The function $f(x)$ just constructed is not the only one which satisfies (4). We can see immediately that every $g(x)=a f(x)+b$ satisfies (4) too-but no other function. Because if

$$
\begin{gathered}
f^{-1}\left(\frac{f(x)+f(y)}{2}\right)=g^{-1}\left(\frac{g(x)+g(y)}{2}\right) ; \quad f(x)=u, \quad f(y)=v, \\
g f^{-1}\left(\frac{u+v}{2}\right)=\frac{g f^{-1}(u)+g f^{-1}(v)}{2} .
\end{gathered}
$$

This is Jensen's equality for the function $g f^{-1}(t)$, satisfied only by $g f^{-1}(t)=a t+b, g(x)=a f(x)+b .6$ The $f(x)$ we constructed is determined by $f(\alpha)=0, f(\beta)=1$.

\section{Nonsymmetric means.}

THEOREM. If the function of two variables $M(x, y)$ satisfies the following conditions (cf. $\$ 1)(\alpha \leqq x, y \leqq \beta)$ :

(i') Strict monotony;

(ii') Continuity;

(iii') Bisymmetry;

(iv') Reflexivity,

then and only then there exists a continuous increasing function $f(x)$ and a real number $0<p<1$ by which $M(x, y)$ has the form $(p+q=1)$ :

$$
M(x, y)=f^{-1}[p f(x)+q f(y)] .
$$

The necessity of the conditions is evident. To prove the sufficiency we construct a symmetric mean $m(x, y)$ satisfying the conditions (i), (ii), (iii), (iv), (v) of $\$ 1$ so that $m(x, y)=f^{-1}((f(x)+f(y)) / 2)$ and we shall show that this $f(x)$ figures in (10).

We obtain $m(x, y)$ as the limit of the following process:

$$
\begin{array}{ll}
\bar{x}_{0}=\min (x, y) & \bar{y}_{0}=\max (x, y), \\
\bar{x}_{1}=\min [M(x, y), M(y, x)], & \bar{y}_{1}=\max [M(x, y), M(y, x)],
\end{array}
$$

$$
\bar{x}_{n+1}=\min \left[M\left(\bar{x}_{n}, \bar{y}_{n}\right), M\left(\bar{y}_{n}, \bar{x}_{n}\right)\right], \bar{y}_{n+1}=\max \left[M\left(\bar{x}_{n}, \bar{y}_{n}\right), M\left(\bar{y}_{n}, \bar{x}_{n}\right)\right],
$$


The sequences $\bar{x}_{n}$ and $\bar{y}_{n}$ are monotonous and bounded and so they have limits $\xi=\lim _{n \rightarrow \infty} \bar{x}_{n}, \eta=\lim _{n \rightarrow \infty} \bar{y}_{n} . \xi=\eta$, for (from $\S 1$ ) $M(x, y)$ is $k$-symmetric (in the weaker sense) and thus if, for example, $\xi<\eta$

$$
\begin{aligned}
\xi & =\lim \bar{x}_{n+1}=\lim \min \left[M\left(\bar{x}_{n}, \bar{y}_{n}\right), M\left(\bar{y}_{n}, \bar{x}_{n}\right)\right] \\
& =\min [M(\xi, \eta), M(\eta, \xi)]>\xi
\end{aligned}
$$

that is, $\tau=\xi=\eta=\lim \bar{x}_{n}=\lim \bar{y}_{n}=m(x, y)$. Also we see now that

$$
\begin{array}{rlrl}
m(x, y) & =\lim x_{n}=\lim y_{n} ; \quad x_{0}=x, \quad y_{0}=y ; \\
x_{1} & =M(x, y), & y_{1} & =M(y, x) ; \cdots ; \\
x_{n+1} & =M\left(x_{n}, y_{n}\right), & y_{n+1} & =M\left(y_{n}, x_{n}\right) ; \cdots .
\end{array}
$$

We prove that this $m(x, y)$ is a mean value satisfying the conditions (i), (ii), (iii), (iv), (v) of §1. (iv) and (v) is fulfilled evidently by (11). To prove (i), (ii), (iii) let us consider first the function $\phi(t ; x, y)$ $=M[M(t, x), M(y, t)] ; \phi(t ; t, t)=t . \phi(t ; x, y)$ is increasing and continuous in $t, x, y$.

$$
\begin{aligned}
\phi(t ; x, y) & =\phi[t ; M(x, y), M(y, x)]=\phi\left(t ; x_{1}, y_{1}\right)=\cdots \\
& =\phi\left(t ; x_{n}, y_{n}\right)=\cdots=\phi(t ; \tau, \tau) \\
& =\phi[t ; m(x, y), m(x, y)]
\end{aligned}
$$

because $M(x, y)$ is $k$-symmetric (in the weaker sense). So the functional equation $t=\phi(t ; x, y)$ is equivalent with

$$
t=\phi(t ; \tau, \tau)=\phi[t ; m(x, y), m(x, y)] .
$$

(13) is satisfied by $t=\tau=m(x, y)$ and this is its only solution, because if, for example, $t<\tau$ would be a solution too, $t=\phi(t ; x, y)=\phi(t ; \tau, \tau)$ $>\phi(t ; t, t)=t$. This is absurd!

Now we prove (i): if $x^{\prime}>x$ then, in (12), $x_{1}^{\prime}>x_{1}, y_{1}^{\prime}>y_{1}, \cdots, x_{n}^{\prime}$ $>x_{n}, y_{n}^{\prime}>y_{n}, \cdots, t^{\prime} \geqq t$. But $t^{\prime}=t$ is impossible, because from $t^{\prime}=t$ and (13), $t=t^{\prime}=\phi\left(t^{\prime} ; x^{\prime}, y\right)=\phi\left(t ; x^{\prime}, y\right)>\phi(t ; x, y)=t$. This is absurd, and thus $m(x, y)$ is increasing: $m\left(x^{\prime}, y\right)>m(x, y)$. The continuity (ii) can be proved as follows: Let $\xi^{(n)}$ be an increasing sequence converging to $x, \eta^{(n)}$ one converging to $y$. The sequence $\tau^{(n)}=m\left(\xi^{(n)}, \eta^{(n)}\right)$ being increasing and bounded converges too: $\tau^{(n)} \rightarrow \bar{\tau}$. If we take the limit of $\tau^{(n)}=\phi\left(\tau^{(n)} ; \xi^{(n)}, \eta^{(n)}\right)$ we have $\bar{\tau}=\phi(\bar{\tau} ; x, y)$. This is only possible if $\bar{\tau}=\tau=m(x, y)$. This and (i) gives (ii). Also the bisymmetry (iii) follows easily from (13) and the $k$-symmetry of $M(x, y)$. (iii) can be deduced also from the equation (15). Thus

$$
m(x, y)=f^{-1}\left(\frac{f(x)+f(y)}{2}\right)
$$


by the Theorem of $\$ 1$. Here $f(x)$ is increasing continuous, and $f(\alpha)=0, f(\beta)=1$.

We show that this is the $f(x)$ which figures in (1). We prove first

$$
M\left[m\left(x_{11}, x_{12}\right), m\left(x_{21}, x_{22}\right)\right]=m\left[M\left(x_{11}, x_{21}\right), M\left(x_{12}, x_{22}\right)\right]
$$

or with another notation: $M[m(x, y), m(u, v)]=m[M(x, u), M(y, v)]$. This is a consequence of (12) and the $k$-symmetry of $M(x, y)$. We write:

$$
\begin{aligned}
& M\left(x_{0}, u_{0}\right)=M(x, u)=s=s_{0}, \quad M\left(y_{0}, v_{0}\right)=M(y, v)=t=t_{0}, \\
& M\left(x_{1}, u_{1}\right)=M[M(x, y), M(u, v)]=M[M(x, u), M(y, v)]=M(s, t)=s_{1}, \\
& M\left(y_{1}, v_{1}\right)=M(t, s)=t_{1}, \\
& M\left(x_{n}, u_{n}\right)=M\left(s_{n-1}, t_{n-1}\right)=s_{n}, \quad M\left(y_{n}, v_{n}\right)=M\left(t_{n-1}, s_{n-1}\right)=t_{n}, \\
& M[m(x, y), m(u, v)]=m(s, t)=m[M(x, u), M(y, v)] \\
& \left(m(x, y)=\lim x_{n}=\lim y_{n}, m(u, v)=\lim u_{n}=\lim v_{n}, m(s, t)=\lim s_{n}=\lim t_{n}\right) . \\
& \text { Substituting (14) into (15) and writing } f\left\{M\left[f^{-1}(\xi), f^{-1}(\eta)\right]\right\} \\
& =F(\xi, \eta) \text { we have: }{ }^{7} \\
& M\left\{f^{-1}\left(\frac{f\left(x_{11}\right)+f\left(x_{12}\right)}{2}\right), f^{-1}\left(\frac{f\left(x_{21}\right)+f\left(x_{22}\right)}{2}\right)\right\} \\
& =f^{-1}\left\{\frac{f\left[M\left(x_{11}, x_{21}\right)\right]+f\left[M\left(x_{12}, x_{22}\right)\right]}{2}\right\}, \\
& F\left(\frac{z_{11}+z_{12}}{2}, \frac{z_{21}+z_{22}}{2}\right)=\frac{F\left(z_{11}, z_{21}\right)+F\left(z_{12}, z_{22}\right)}{2}
\end{aligned}
$$

$(f(x)=z)$. This is Jensen's equality ${ }^{8}$ for functions of two variables, the only solution of which is the linear function $f\left\{M\left[f^{-1}(\xi), f^{-1}(\eta)\right]\right\}$ $=F(\xi, \eta)=p \xi+q \eta+r$,

$$
f[M(x, y)]=p f(x)+q f(y)+r
$$

$(\xi=f(x), \eta=f(y))$. If we put here $x=y=\alpha$ we have $r=0$; if $x=y=\beta$, $p+q=1$. (By $x=\alpha, y=\beta, f[M(\alpha, \beta)]=q$ and by $x=\beta, y=\alpha, f[M(\beta, \alpha)]$

7 Cf. Aumann, Konvexe Funktionen und die Induktion bei Ungleichungen zwischen Mittelwerten, Bay. Akademie derWissenschaften, Munich, Sitzungsberichte (1933). J. Aczel, $A$ generalization of the notion of convex functions, Norske Videnskabers Selskabs Forhandlingen vol. 19 (1946).

${ }^{8}$ Cf. Hardy, Littlewood, P6lya, Inequalities, pp. 79-80. 
$=p$.$) Thus$

$$
M(x, y)=f^{-1}[p f(x)+q f(y)] \quad(p+q=1) \text { Q.E.D. }
$$

Together with $f(x)$ evidently every $g(x)=a f(x)+b$ satisfies $(10)$ too. These are the only solutions as $m(x, y)$ is uniquely defined by $M(x, y)$ (11) and we have seen in $\$ 1$ that $a f(x)+b$ is the most general function satisfying (14). The weight $p$ is uniquely defined by (10) because if $(p+q=r+s=1)$ :

$$
\begin{aligned}
f^{-1}[p f(x)+q f(y)] & =g^{-1}[r g(x)+s g(y)], \quad w=g(z)=a f(z)+b, \\
z & =g^{-1}(w)=f^{-1}\left(\frac{w-b}{a}\right),
\end{aligned}
$$

$a p f(x)+a q f(y)=a r f(x)+b r+a s f(y)+b s-b$ and thus $p=r, q=s$.

\section{Bisymmetric functions.}

THEOREM. If a function of two variables which we shall write $[x, y]$ is $(\alpha \leqq x, y \leqq \beta ; \alpha \leqq[x, y] \leqq \beta)$ :

( $\left.\mathrm{i}^{\prime \prime}\right)$ Increasing;

(ii') Continuous;

(iii') Bisymmetric: $\left[\left[z_{11}, z_{12}\right],\left[z_{21}, z_{22}\right]\right]=\left[\left[z_{11}, z_{21}\right],\left[z_{12}, z_{22}\right]\right]$, then and only then there exists an increasing continuous function $f(x)$ and three real numbers $r, s, t$, by which

$$
[x, y]=f^{-1}\{r f(x)+s f(y)+t\} .
$$

We prove the theorem by constructing a mean value $(\$ 2) M(x, y)$ $=f^{-1}\{p f(x)+q f(y)\}$ and by showing that this is the $f(x)$ which figures in (16).

We see from $\left(\mathrm{i}^{\prime \prime}\right)$ and $\left(\mathrm{ii}^{\prime \prime}\right)$ that the function $F(z)=[z, z]$ is continuous and increasing. The functions $F^{-1}(z)$ and $F^{2}(z)=F\{F(z)\}$ $=[[z, z],[z, z]]$ are, also. We prove that the function $z=M(x, y)$ $=F^{-1}([x, y])$ is a mean value which satisfies the conditions $\left(\mathrm{i}^{\prime}\right)$, (ii'), (iii'), (iv') of \$2. In fact, $\left(\mathrm{i}^{\prime}\right)$ and (ii') follow from $\left(\mathrm{i}^{\prime \prime}\right)$ and (ii'); as for (iv'): $M(z, z)=F^{-1}([z, z])=F^{-1} F(z)=z$. We have to verify yet (iii'). We write:

$$
\begin{gathered}
{\left[z_{11}, z_{12}\right]=\left[z_{1}, z_{1}\right], z_{1}=M\left(z_{11}, z_{12}\right),\left[z_{11}, z_{21}\right]=\left[\bar{z}_{1}, \bar{z}_{1}\right], \bar{z}_{1}=M\left(z_{11}, z_{21}\right),} \\
{\left[z_{21}, z_{22}\right]=\left[z_{2}, z_{2}\right], z_{2}=M\left(z_{21}, z_{22}\right),\left[z_{12}, z_{22}\right]=\left[\bar{z}_{2}, \bar{z}_{2}\right], \bar{z}_{2}=M\left(z_{12}, z_{22}\right),} \\
{\left[z_{1}, z_{2}\right]=[z, z], \quad z=M\left(z_{1}, z_{2}\right)=M\left\{M\left(z_{11}, z_{12}\right), M\left(z_{21}, z_{22}\right)\right\},} \\
{\left[\bar{z}_{1}, \bar{z}_{2}\right]=[\bar{z}, \bar{z}], \quad \bar{z}=M\left(\bar{z}_{1}, \bar{z}_{2}\right)=M\left\{M\left(z_{11}, z_{21}\right), M\left(z_{12}, z_{22}\right)\right\} .}
\end{gathered}
$$

By applying (iii'") repeatedly we have $z=\bar{z}$, because 


$$
\begin{aligned}
F^{2}(z) & =[[z, z],[z, z]]=\left[\left[z_{1}, z_{2}\right],\left[z_{1}, z_{2}\right]\right]=\left[\left[z_{1}, z_{1}\right],\left[z_{2}, z_{2}\right]\right] \\
& =\left[\left[z_{11}, z_{12}\right],\left[z_{21}, z_{22}\right]\right]=\left[\left[z_{11}, z_{21}\right],\left[z_{12}, z_{22}\right]\right]=\left[\left[\bar{z}_{1}, \bar{z}_{1}\right],\left[\bar{z}_{2}, \bar{z}_{2}\right]\right] \\
& =\left[\left[\bar{z}_{1}, \bar{z}_{2}\right],\left[\bar{z}_{1}, \bar{z}_{2}\right]\right]=[[\bar{z}, \bar{z}],[\bar{z}, \bar{z}]]=F^{2}(\bar{z}), \\
M & \left.M\left(z_{11}, z_{12}\right), M\left(z_{21}, z_{22}\right)\right\}=z=\bar{z}=M\left\{M\left(z_{11}, z_{21}\right), M\left(z_{12}, z_{22}\right)\right\} .
\end{aligned}
$$

Thus we have by the theorem of $\S 2:[x, y]=F\{M(x, y)\}=F f^{-1}\{p f(x)$ $+q f(y)\}=\psi\{p f(x)+q f(y)\} \quad\left(\psi(z)=F f^{-1}(z)\right)$.

We substitute this result into $[[x, y],[x, y]]=[[x, x],[y, y]]$ (this follows from ( $\left.\mathrm{iii}^{\prime \prime}\right)$ ),

$$
\psi(f \psi\{p f(x)+q f(y)\})=\psi(p f \psi f(x)+q f \psi f(y)) .
$$

If we write $f \psi(z)=h(z) ; f(x)=u, f(y)=v$ we have $h(p u+q v)=p h(u)$ $+q h(v)$. This is Jensen's equality ${ }^{9}$ and hence $f \psi(z)=h(z)=\omega z+t$ $(\omega, t$ are constants)

$$
f([x, y])=\omega\{p f(x)+q f(y)\}+t=r f(x)+s f(y)+t \text { Q.E.D. }
$$

As the $M(x, y)$ is uniquely determined by $[x, y]$, all functions $g(x)$ satisfying (16) have the form $g(x)=a f(x)+b$. The "weights" $r, s$ are defined uniquely by $M(x, y)$ but $t$ is not. For if $f^{-1}(r f(x)+s f(y)+t)$ $=g^{-1}(\rho g(x)+\sigma g(y)+\tau), w=g(z)=a f(z)+b, z=g^{-1}(w)=f^{-1}((w-b) / a)$ then $\operatorname{arf}(x)+a s f(y)+a t=a \rho f(x)+b \rho+a \sigma f(y)+b \sigma+\tau-b$ and thus $\rho=r, \sigma=s, \tau=a t+b(1-\rho-\sigma)=a t+b(1-r-s)$. If $r+s \neq 1$ we can choose $b$ so that $\tau=0$, namely $b=a t /(r+s-1)$. Hence for $\phi(x)=f(x)$ $+t /(r+s-1)$ and for every $\psi(x)=a \phi(x)$ (and only for them):

$$
[x, y]=\phi^{-1}\{r \phi(x)+s \phi(y)\} \quad(r+s \neq 1) .
$$

If $[x, y]=x \circ y$ satisfies instead of (iii" $\left.{ }^{\prime \prime}\right)$ the stronger conditions $x \circ(y \circ z)=(x \circ y) \circ z$ and $x \circ y=y \circ x$, that is, if $x \circ y$ is an increasing continuous, "associative, and commutative operation"10 we have, by putting, in (16), $r=s=1$, from (17), the following corollary.

COROLlARY. Every increasing, continuous, associative, and commutative operation has the form

$$
x \circ y=f^{-1}\{f(x)+f(y)+t\}=\phi^{-1}\{\phi(x)+\phi(y)\} .
$$

BUDAPEST, HUNGARY

${ }^{9}$ Hardy, Littlewood, P6́lya, Inequalities, p. 74.

${ }_{10}$ This associativity has naturally nothing to do with the associativity defined for mean values by Kolmogoroff and Nagumo quoted in $\$ 1$. 